\title{
Solidarité et coaching confraternels - répondre aux besoins des médecins rencontrant des difficultés
}

\author{
Le présent texte est la version révisée d'un document établi en 2000, alors que \\ l'auteur était médecin cantonal et avait eu à connaître au cours des années \\ des situations de confrères rencontrant des problèmes sérieux dans leur santé \\ et/ou dans leur vie professionnelle. Situations qui ont pu donner lieu à des \\ issues hautement regrettables qu'il faudrait pouvoir prévenir de manière \\ efficace mais où cela ne s'avère pas aisé. Où il importe par conséquent de faire \\ appel à l'intelligence et à la disponibilité du corps professionnel, dans l'opti- \\ que du projet ReMed développé par la FMH [1, 2].
}

\section{Jean Martin*}

*'auteur est ancien médecin cantonal du canton de Vaud.

a Il n'y a pas lieu de cacher que, sur ce dernier point, la supervision de la pratique n'a rien d'aisé. L'essentiel de ce qui se passe entre soigné et soignant l'est dans un cadre très privé, où la confidentialité est hautement valorisée et où les faits ne sont pas toujours documentés. On n'étonnera personne en disant que le patient qui formule des griefs, ou l'autorité de surveillance qui se renseigne, ont souvent des difficultés à rassembler des éléments objectifs appropriés. Pas rarement, on est dans une situation de type «la parole de l'un contre la parole de l'autre», qui ne permet usuellement pas de conclure.

Correspondance:

Dr Jean Martin, p.-d.

La Ruelle 6

CH-1026 Echandens

jean.martin@urbanet.ch
On se souvient de la parole biblique «Suis-je le gardien de mon frère?». On y fait référence négativement parfois, considérant que nous sommes dans un monde d'adultes responsables et que ce n'est pas à nous, le cas échéant, de prendre la main de notre prochain en situation lourde ou délicate. Toutefois, la médecine est un métier difficile, source de surcharge, de tensions diverses, parfois de tentations, susceptibles de déstabiliser le praticien. Il importe alors que ce dernier ne se retrouve pas seul mais qu'il sache où et comment il peut trouver conseil et soutien, en particulier auprès de confrères.

\section{Le cadre}

L'autorité sanitaire cantonale (département chargé de la santé et en son sein le service de la santé publique) est amenée à enregistrer des plaintes ou des situations préoccupantes concernant la pratique de professionnels. Des questions sont posées par des patients inquiets ou insatisfaits, de diverses manières des incidents sont portés à notre connaissance, y compris par l'autorité judiciaire.

Ces situations ne sont agréables pour personne. Il reste qu'elles surviennent, demandent que soit vérifiée la capacité de pratiquer de ces personnes et doivent être évaluées en fonction notamment des aspects suivants:

- la nécessaire supervision de la qualité de la pratique des professionnels de la santé et la protection des patients qui se confient à ces derniers, mission majeure du Département. Pour l'Etat, de même sans doute que pour les corporations professionnelles - et du point de vue de la population quand elle s'exprime à ce sujet -, cette tâche doit aujourd'hui être accomplie avec autant de précision que nécessaire, sans complaisance. Dans le respect des grandes règles de l'action publique que sont la légalité, l'égalité de traitement (à affaires comparables, conclusions et décisions comparables) et la proportionnalité (notion selon laquelle les mesures prises doivent être dans un rapport adéquat avec la gravité des faits);

- dans une société libérale, les garanties de ne pas être sanctionné sur des bases fragiles ou insuffisantes, tant qu'il n'a pas été démontré qu'on n'est pas (ou plus) capable d'exercer sa profession. $^{\mathrm{a}}$

\section{La difficulté de vivre des circonstances qui mettent en cause sa pratique professionnelle}

Il n'est pour le moins pas agréable et il peut être traumatisant, pour un professionnel, d'être interpellé à propos de faits où sa compétence ou son comportement sont mis en cause, par un patient insatisfait, par le médecin cantonal ou une instance mandatée - le Conseil de santé a pour rôle dans le canton de Vaud de préaviser vis-à-vis du chef de département en matière de mesures disciplinaires. Bien qu'une évolution certaine se soit marquée au cours des vingt dernières années, 
ce n'est pas toujours facilement que le médecin - ou autre professionnel - accepte que soit exercé le droit de poser n'importe quelle question à n'importe qui. (NB: nous avons souvent employé cette formule: rien en effet ne saurait empêcher de poser des questions, cela ne veut cependant pas dire qu'on aura toujours une réponse satisfaisante - selon les cas, la personne questionnée pourrait ne pas avoir le droit de répondre ou garde la liberté de décliner de le faire.) Il est tout à fait important de ne pas considérer que des demandes d'explications ou d'autres interrogations correspondent forcément à un manque de confiance, une suspicion indue ou la manifestation d'un caractère revendicateur. Ceux qui comme moi étaient assistants dans les années 1960 se souviennent que, à l'époque, il était rare qu'un malade, aussi hardi soit-il, pose des questions critiques au médecin - les temps ont changé et, pour l'essentiel, le changement est justifié.

La réalité est que, parfois, on a pu effectivement être inadéquat. C'est difficile à vivre à plusieurs égards:

- au plan des conséquences possibles s'agissant de responsabilité civile voire pénale;

- du point de vue de l'image de soi (vis-à-vis de soi-même et vis-à-vis d'autres, proches, collègues, etc.);

- cela l'est surtout vis-à-vis du patient quand une prise en charge a été insatisfaisante et que ce dernier en a pâti. Il importe de souligner qu'il n'y a pas que des actes du registre technique ou opératoire qui entrent ici en ligne de compte; certaines inadéquations dans le dialogue avec le patient ou quant au respect qu'on lui doit correspondent à de la «malpratique» (le domaine des paroles, attitudes et gestes à connotation sexuelle n'étant qu'un type de telles situations).

\section{Les faits}

Dans leur froide objectivité, les faits que nous avons dû enregistrer au cours des années 1990 sont que quatre médecins et un membre d'une autre profession de la santé sont décédés pendant une période où ils étaient en relation avec le Service de la santé publique pour des problèmes mettant en cause leur capacité à pratiquer - pour des motifs à dimension disciplinaire ou d'altération de leur état de santé. Ils avaient entre 40 et 60 ans.

Il ne peut évidemment s'agir ici de décrire ces situations en détail. On peut relever toutefois que, dans plusieurs cas, il y avait un problème de consommation inadéquate de substances (drogues illégales, alcool) et/ou une problémati- que psychiatrique; deux personnes souffraient d'affections somatiques sérieuses. S'agissant de l'issue funeste, elle était due une fois à un accident de la route et deux fois à une auto-agression manifeste. Dans deux autres cas, il y a eu mort subite inattendue dont la raison précise ne nous est pas connue.

\section{Le besoin d'agir - comment?}

Si de telles observations sont préoccupantes, on reste dans une large ignorance d'éventuelles relations de cause à effet. ${ }^{b}$ Indépendamment de démarches extérieures, ces professionnels étaient des personnes fragilisées; l'altération de leur capacité de pratiquer (avérée) était une facette d'une situation personnelle - et touchant l'entourage - grave, à haut risque d'accident. Mais cela ne supprime pas la pertinence de se demander si, par un encadrement et un soutien appropriés, on aurait pu éviter ces issues; y compris le cas échéant en faisant admettre la nécessité d'une diminution ou interruption de la pratique (temporaire ou définitive), pour soulager la tension, prendre du recul, augmenter les chances de se rétablir.

A l'évidence, l'autorité sanitaire et le médecin cantonal en son sein sont ici dans une position délicate [2]. Interpellés sur des situations posant problème, ils sont chargés de les instruire et de poser des questions parfois désagréables, susceptibles d'être ressenties comme une atteinte à l'estime et l'image de soi du professionnel. Leur rôle est parfois comparable à celui d'un procureur. Le fait de s'impliquer activement dans le conseil ou l'accompagnement du professionnel en difficulté entraîne un conflit d'intérêts.

Il y avait généralement dans ces cas des confrères médecins traitants. Relevons que, comme une solution intermédiaire, a pu être posé comme condition à la poursuite de la pratique que le professionnel soit régulièrement suivi par un thérapeute, ce dernier étant de manière anticipée délié du secret médical en vue de nous informer si la situation se péjorait et ne permettait plus une pratique adéquate. On constate par ailleurs que ces médecins traitants privés eux aussi éprouvent des difficultés à obtenir de la personne en difficulté une discipline thérapeutique et un suivi adéquats.

Comment établir un filet de sécurité? Comment stimuler des démarches de solidarité et de coaching? Etant entendu qu'un premier geste, si l'un d'entre nous prend conscience de la situation délicate d'un confrère (qu'il s'agisse d'un ami personnel, d'un camarade d'étude, d'un collègue de la même discipline ou d'un membre du même club hors du sérail médical), est de documents y relatifs). Il apparaît que les démarches entreprises ont été strictement adéquates.
Nous avons de notre côté réexaminé attentivement les dossiers en question (et, dans un cas, l'avocat-conseil d'une famille a 
l'approcher avec le doigté voulu et d'entamer le dialogue.

Dans l'élaboration d'un dispositif organisé de soutien, la corporation est à notre sens la mieux placée, la mieux armée (ou la «moins mal» placée - la gestion de ces cas restera toujours délicate). Ainsi la FMH dans un cadre tel que le projet ReMed, les sociétés cantonales, le cas échéant les sociétés de spécialistes ou généralistes. Une vocation traditionnelle des associations professionnelles est d'assister les membres en difficulté au plan personnel, pécuniaire ou autre.

Il s'agit d'imaginer un système, un réseau, qui de façon non indûment intrusive permette et assure un contact avec la personne en difficulté; ceci en vue de l'aider pratiquement et dans les débats intérieurs que ne peuvent manquer de créer des problèmes psychosociaux ou somatiques graves et la remise en cause de sa pratique. Nous n'avons pas de modèle tout fait à proposer. Le rappel ci-dessus de nos observations et préoccupations a pour but de confirmer la pertinence et le besoin, sans délai, d'entreprendre, de mettre en place quelque chose de suffisamment étoffé et d'accès aisé: certains d'entre nous, aux prises avec une activité astreignante, dans une société en mutation rapide, rencontrent des difficultés majeures et il importe qu'ils puissent bénéficier d'aide.

De plus, peut-être faut-il que, en général, nous soyons sensibilisés à l'idée que, pour chacun, les soucis graves, professionnels ou personnels, psychologiques ou physiques, cela peut arriver. Et qu'il convient d'intégrer à l'avance - de se convaincre - qu'il est alors permis (plus que cela, qu'il est légitime et souhaitable) de se tourner assez tôt vers une ou des personnes de confiance.

\section{Références}

1 Hersperger M, Peltenburg M. ReMed: un réseau d'assistance pour les membres de la FMH. Bull Méd Suisses. 2006;87(25):1159-60.

2 Martin J. Un réseau tel que ReMed: initiative judicieuse, bienvenue, nécessaire. Bull Méd Suisses. 2006;87(27/28):1260. 